\title{
Modelling and Simulation for Concrete Durability: Mechanism and Prediction
}

\author{
Qing-feng Liu $\mathbb{D},{ }^{1}$ Branko Šavija $\mathbb{D}^{2},{ }^{2}$ Jin Xia $\mathbb{D}^{3},{ }^{3}$ Xiaoshan Lin $\mathbb{D}^{4},{ }^{4}$ and Dongshuai Hou $\mathbb{D}^{5}$ \\ ${ }^{1}$ State Key Laboratory of Ocean Engineering, School of Naval Architecture, Ocean and Civil Engineering, Shanghai Jiao \\ Tong University, Shanghai 200240, China \\ ${ }^{2}$ Microlab, Faculty of Civil Engineering and Geosciences, Delft University of Technology, Delft 2628CN, Netherlands \\ ${ }^{3}$ Institute of Structural Engineering, Zhejiang University, Hangzhou, China \\ ${ }^{4}$ School of Engineering, RMIT University, Australia \\ ${ }^{5}$ Department of Civil Engineering, Qingdao University of Technology, China
}

Correspondence should be addressed to Qing-feng Liu; liuqf@sjtu.edu.cn

Received 28 July 2021; Accepted 28 July 2021; Published 16 August 2021

Copyright () 2021 Qing-feng Liu et al. This is an open access article distributed under the Creative Commons Attribution License, which permits unrestricted use, distribution, and reproduction in any medium, provided the original work is properly cited.

Concrete is the most widely used man-made material in the modern construction industry. However, the service life of concrete has been seriously shortened due to durability problems, particularly when serving in nonideal environments and suffering internal/external attacks. In this regard, the development of a better understanding of the deterioration mechanisms as well as reliable prediction methods for durability properties and/or long-term performance of concrete is necessary. Modern computational modelling theories and methods are favorable for bringing innovative solutions to the above issues. The objective of this special issue is to present novel modelling studies on concrete durability by using analytical/numerical approaches alone or in conjunction with experimental techniques.

During the submission period, we received more than 20 submissions from more than 10 different countries. All these submissions were well written and had their own innovations, but only 4 of them were selected as they were closer to the subjects of this special issue. The articles published in this special issue covered various scales from the C-S-H to reinforced concrete components and finally concrete-based buildings, and the following topics were involved: (a) simulation of deterioration processes; (b) permeability and diffusivity modelling; (c) cracking and fracture detection; (d) simulation of rebar corrosion process; (e) simulation of hydration processes; and ( $\mathrm{f}$ ) prediction model of long-term performance. The contributions offered by each paper are detailed in the following.

The paper entitled "Numerical Study on Diffusion of Chloride and Induced Rebar Corrosion by Two-Dimensional Multiscale Approach" by X. Tu et al. described a twodimensional multiscale model including core parts and compensation apart and three-phase mesoscales including binder, aggregate, and ITZ to simulate chloride diffusion within concrete. The diffusivity and width of ITZ, volume fraction, and grade of aggregate were studied. Besides, the authors also discussed rebar corrosion in terms of intrusion by external chloride. The distribution of chloride content was considered with the change of ITZ and compensation. This presented work showed the benefits of a multiscale model and provided the basis for future large-scale structure numerical studies.

The paper entitled "Modeling of the Axial Load Capacity of RC Columns Strengthened with Steel Jacketing under Preloading Based on FE Simulation" by A. M. Sayed and H. M. Diab devised a macroscale three-dimensional finite element (FE) model to analyze the conduct and capacity of RC (reinforced concrete) square columns reinforced with steel jacketing under static preload. In comparison with previously experimental results, the proposed $\mathrm{FE}$ model of the component showed good accuracy. Thus, the model revealed the potential for calculating RC columns' load capacity and for predicting accurate failure modes. 
The paper entitled "Hydration of Early Age Cement Paste with Nano- $\mathrm{CaCO}_{3}$ and SAP by LF-NMR Spectroscopy: Mechanism and Prediction" by $\mathrm{H}$. Zhao et al. brought a deep-seated mechanism such as the interaction among various mineral hydrations into consideration and tested the evolution of the physically bound water using the low-field nuclear magnetic resonance (LF-NMR) technology. The microscope hydration process was divided into 4 periods and analyzed, respectively. The effects of water to cement ratio $(w / c)$, $\mathrm{NC}$ (nano- $\mathrm{CaCO}_{3}$ ), and SAP (superabsorbent polymer) on each hydration process period of cementbased materials were discussed in detail. Meanwhile, a modified hydration model considering the content of NC and SAP is also proposed.

The paper entitled "Improved Crack Detection and Recognition Based on Convolutional Neural Network" by K. Chen et al. improved crack detection based on convolutional neural networks, which can automatically detect whether an image contains cracks and mark the location of the cracks. In this paper, the authors firstly tested and improved the convolution neural networks with an accuracy of $99.71 \%$. Secondly, they introduced this automatic system for historic buildings. From their experiments, the system had a certain value for periodic inspection and maintenance of long-term buildings.

With this special issue, we hope researchers (and interested readers as well) who have been engaged in this or related areas receive advanced information, gain ideas, and then further promote the development of modelling of concrete durability. We, the guest editors, hope it will stimulate relevant research interests on both performance prediction at the structural level and mechanism investigations at the material level in the coming future.

\section{Conflicts of Interest}

We declare that we have no financial or personal relationships with other people or organizations that can inappropriately influence the work included in this special issue.

Qing-feng Liu

Branko Šavija Jin Xia

Xiaoshan Lin

Dongshuai Hou 